\title{
Archéopages
}

Archéopages

Archéologie et société

46 | 2018

Maisons

\section{La maison rurale en Sologne bourbonnaise et en Basse-Auvergne au Moyen Âge}

Les limites d'une définition typochronologique

Rural houses in Sologne bourbonnaise and Basse-Auvergne in the Middle Ages.

The limits of a typochronological definition

La vivienda rural en la Sologne del Borbonés y en la Baja Auvernia durante la

Edad Media. Los límites de una definición tipocronológica

Sébastien Gaime, Julie Charmoillaux et Daniel Parent

\section{OpenEdition}

Journals

Édition électronique

URL : https://journals.openedition.org/archeopages/4079

DOI : 10.4000/archeopages.4079

ISSN : 2269-9872

Éditeur

INRAP - Institut national de recherches archéologiques préventives

Édition imprimée

Date de publication : 1 décembre 2018

Pagination : 48-59

ISSN : 1622-8545

Référence électronique

Sébastien Gaime, Julie Charmoillaux et Daniel Parent, « La maison rurale en Sologne bourbonnaise et en Basse-Auvergne au Moyen Âge ", Archéopages [En ligne], 46 | 2018, mis en ligne le 01 décembre 2020, consulté le 02 juin 2021. URL : http://journals.openedition.org/archeopages/4079 ; DOI : https:// doi.org/10.4000/archeopages.4079 


\title{
La maison rurale en Sologne
}

\section{bourbonnaise et en}

\section{Basse-Auvergne au Moyen Âge}

Les limites d'une définition

typochronologique

\author{
Sébastien Gaime Inrap, UMR 6042 "GEOLAB" \\ Julie CharmoillauX Ministère de la Culture, UMR 5648 « Iham» \\ Daniel Parent Inrap
}

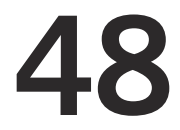

1. Cet article présente les principales conclusions

d'un travail collectif de recherche, réalisé entre 2009 et 2016 , réunissant une équipe d'archéologues et

d'historiens (SRA, Inrap, SAMDA, université Rennes II, université Lyon II, Hadès, Hévéa) sous la double coordination des auteurs.

À paraître en 2019

dans la collection

« Recherches

archéologiques».
Pour l'archéologue, parler de maison est une gageure ; en effet les données archéologiques, et sans doute plus encore en rural qu'en urbain, sont très limitées, avec une conservation de quelques dizaines de centimètres au-dessus du sol naturel le plus souvent. Il n'est pas toujours aisé de caractériser précisément les types de vestiges, ni les fonctions exactes des structures fouillées, ni le statut social des habitants. Si le caractère paysan est sous-entendu le plus souvent dans la littérature archéologique par les termes habitat rural, la réalité est complexe et multiple : toutes les classes sociales habitent la campagne, paysans, artisans, religieux, nobles et tous ceux que l'on nomme actuellement élites. De plus, chaque classe sociale est elle-même hétérogène.

La maison peut se définir comme le lieu d'habitation quotidienne pour l'homme : on s'y abrite des intempéries, de la nuit, des animaux voire des hommes ; on y dort, fait la cuisine et parfois on y travaille. Généralement, un bâtiment est identifié comme maison d'habitation un peu par défaut, lorsqu'il possède au moins quatre murs périphériques fermant l'espace. L'existence d'un sol d'occupation parsemé de vestiges d'activités dites domestiques (tessons de céramique, faune, quelques objets...) est aussi un argument recevable pour restituer l'emprise de la maison. Ce critère d'identification d'une maison est celui des archéologues. Pour les historiens ${ }^{\mathbf{1}}$, il en est autrement : le feu caractérise l'habitation puisque le terme, par glissement sémantique, aboutit à désigner la maison dans son ensemble dès l'époque romaine, puis la structure familiale paysanne à partir du XIII ${ }^{\mathrm{e}}$ siècle (Pesez, 1986) : " pour recenser les habitants qui composent un hameau ou un village, les documents fiscaux comptabilisent les familles où l'on fait du feu. » (Sirot, 2011, p. 33).

Toutefois, dans le corpus archéologique étudié [ill. 1a et b], seuls 40 des 108 bâtiments possèdent des foyers... S'agit-il d'une preuve rédhibitoire que les autres bâtiments ne sont pas des maisons ? Cela n'est pas certain. D'abord pour des problèmes évidents de conservation des vestiges : le foyer, de même que les murs ou les sols, n'est pas toujours conservé. Ensuite, il y a sans doute des moyens autres que le foyer, stricto sensu, pour disposer de flammes, lesquels ne laissent pas ou peu de traces archéologiques (foyers surélevés, braseros, etc.). Des sols chargés en charbons de bois témoignent de la proximité de foyers, quand bien même la structure de chauffe n'est pas archéologiquement identifiée. Enfin, il faut aussi distinguer les foyers à vocation artisanale de ceux qui relèvent d'une fonction domestique, même si les deux fonctions sont parfois réunies. Nous avons donc tenté de repérer les autres critères - éléments architecturaux et aménagements intérieurs-qui, en archéologie, pourraient permettre de distinguer la maison des autres bâtiments.

\section{Les éléments architecturaux}

Pour l'archéologue, la manière d'habiter se perçoit avant tout par la manière de construire. Ce sont donc les techniques architecturales qui vont aider à définir la maison rurale médiévale : l'implantation dans le sol, la structure porteuse des constructions, le plan, les dimensions, les matériaux de construction et les toitures.

\section{L'implantation dans le sol et les structures} porteuses

Parmi les 108 bâtiments répertoriés, on distingue quatre types d'implantation dans le sol : 


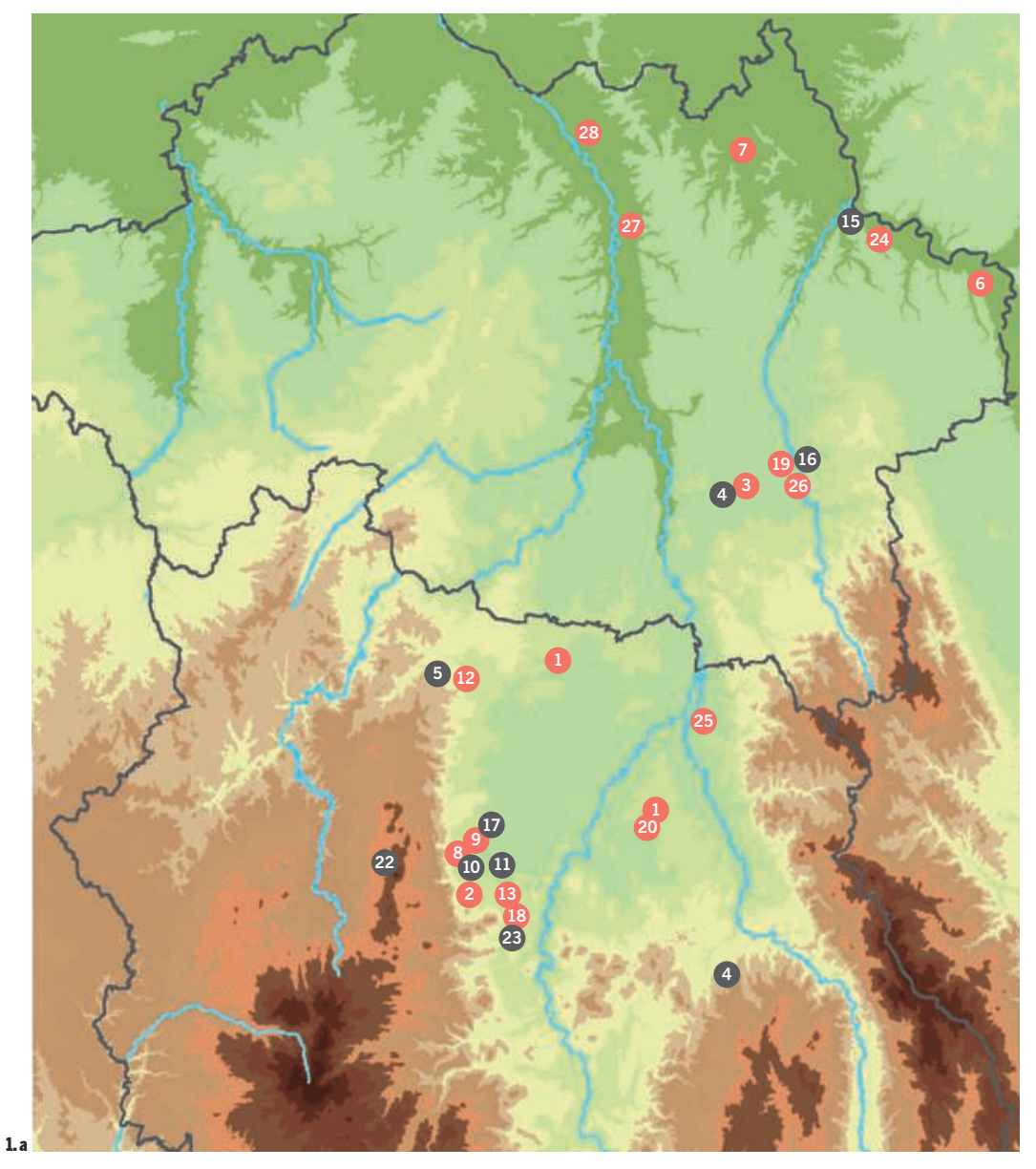

\begin{tabular}{|c|c|}
\hline $138-250 \mathrm{~m}$ & 1.a.L'étude s'est concentrée \\
\hline $251-350 \mathrm{~m}$ & sur les deux entités \\
\hline $351-450 \mathrm{~m}$ & $\begin{array}{l}\text { géographiques que sont la } \\
\text { Grande Limagne et la Sologne }\end{array}$ \\
\hline $451-600 \mathrm{~m}$ & bourbonnaise. Les 18 sites \\
\hline $601-700 \mathrm{~m}$ & archéologiques étudiés \\
\hline $701-800 \mathrm{~m}$ & $\begin{array}{l}\text { S'Implanitent dans des zonies } \\
\text { de plaines et de plateaux, à }\end{array}$ \\
\hline $801-900 \mathrm{~m}$ & moins de $500 \mathrm{~m}$ d'altitude (ce \\
\hline $901-1000 \mathrm{~m}$ & qui exclut les édifices de \\
\hline $1001-1150 \mathrm{~m}$ & $\begin{array}{l}\text { moyenne montagne) et } \\
\text { s'échelonnent du ve au }\end{array}$ \\
\hline $1151-1300 \mathrm{~m}$ & xve siècle. Sur les 108 édifices \\
\hline $1301-1500 \mathrm{~m}$ & du corpus, 55 appartiennent \\
\hline $1501-1874 \mathrm{~m}$ & $\begin{array}{l}\text { dans le Puy-de-Dôme, et } 27 \\
\text { dans l'Allier) et } 53 \text { sont datés } \\
\text { du second Moyen Âge } \\
\text { (40 bâtiments dans le }\end{array}$ \\
\hline site du corpus général & Puy-de-Dôme et 13 dans \\
\hline limite de département & $\begin{array}{l}\text { 1b. La répartition des } \\
\text { bâtiments entre les sites est } \\
\text { assez irrégulière; par } \\
\text { exemple, le site des Colis } \\
\text { compte } 18 \text { bâtiments et celui } \\
\text { de la Cabane, deux. }\end{array}$ \\
\hline
\end{tabular}

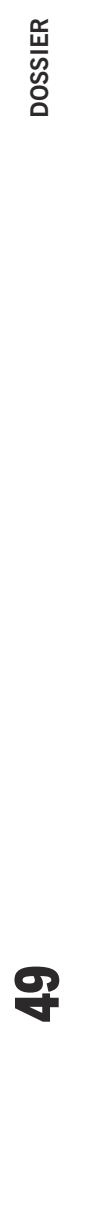

\begin{tabular}{|c|c|c|}
\hline COMMUNE & NOM SITE & PÉRIODE \\
\hline 1. Aigueperse (63) & Cérais (les) & $\mathrm{VIII} / \mathrm{X}^{\mathrm{e}}-\mathrm{XI}^{\mathrm{e}} / \mathrm{XIII}^{\mathrm{e}}$ \\
\hline 2. Beaumont (63) & Mouraude (la) & $\overline{\mathrm{XIII}} \mathrm{e}^{\mathrm{e}}$ \\
\hline 3. Billezois (о3) & Falconnière & $\mathrm{XIV}^{\mathrm{e}-\mathrm{XV} \mathrm{e}^{\mathrm{e}}}$ \\
\hline 4. Cellioux (63) & Chamsolier & $\mathrm{xIV}^{\mathrm{e}}$ \\
\hline 5. Charbonnieres-les-Vieilles (63) & Chauviat & $\mathrm{XIV}^{\mathrm{e}}$ \\
\hline 6. Chassenard (o3) & Génerie (la) & $\mathrm{x}^{\mathrm{e}}$ \\
\hline 7. Chevagnes (оз) & Tronçais (le) & $\mathrm{XIII}^{\mathrm{e}}-\mathrm{XIV}^{\mathrm{e}}$ \\
\hline 8. Clermont-Fd (63) & Bouys & $\overline{\mathrm{VIII}^{\mathrm{e}}-\mathrm{XII}^{\mathrm{e}}}$ \\
\hline 9. Clermont-Fd (63) & Croix De Neyrat & $\mathrm{X}^{\mathrm{e}}-\mathrm{XII}^{\mathrm{e}} / \mathrm{XIII}^{\mathrm{e}}-\mathrm{XIV}^{\mathrm{e}}$ \\
\hline 10. Clermont-Fd (63) & Carmes (les) & $\mathrm{XIV}^{\mathrm{e}}$ \\
\hline 11. Clermont-Fd (63) & Rue Elisée Reclus & $\mathrm{XII}^{\mathrm{e}}-\mathrm{XIII}^{\mathrm{e}}$ \\
\hline 12. Combronde (63) & Cruchades (les) & $\mathrm{XIII}^{\mathrm{e}}$-XIV ${ }^{\mathrm{e}}$ \\
\hline 13. Cournon (63) & Bois Joli (le) & $\mathrm{VII}^{\mathrm{e}}$ \\
\hline 14. Creuzier le Neuf (o3) & Ancizes (les) & XIII $^{\mathrm{e}}-\mathrm{XIV}^{\mathrm{e}}$ \\
\hline 15. Diou (o3) & Bois Prat (le) & $\mathrm{XI}^{\mathrm{e}}-\mathrm{XIII}^{\mathrm{e}}$ \\
\hline 16. Droiturier (оз) & Grand Remblai (le) & VIII $^{\mathrm{e}}-\mathrm{x}^{\mathrm{e}}$ \\
\hline 17. Gerzat & Marterol & $\mathrm{XII}^{\mathrm{e}-\mathrm{XIII}}{ }^{\mathrm{e}}$ \\
\hline 18. La Roche Blanche / Orcet (63) & Enfer $\left(l^{\prime}\right)$ & $\mathrm{VI}^{\mathrm{e}}-\mathrm{VIII}^{\mathrm{e}}$ \\
\hline 19. Lapalisse(о3) & Bel Air & $\mathrm{VI}^{\mathrm{e}}-\mathrm{VII}{ }^{\mathrm{e}} / \mathrm{IX}^{\mathrm{e}}-\mathrm{X}^{\mathrm{e}}$ \\
\hline 20. Lezoux (63 & Bombènes-Parking Du Musée (les) & $\mathrm{XI}^{\mathrm{e}}-\mathrm{XII}^{\mathrm{e}}$ \\
\hline 21. Lezoux (63) & Mon Repos & $\mathrm{XI}^{\mathrm{e}}-\mathrm{XII}^{\mathrm{e}}$ \\
\hline 22. Olby (63) & Motte (la) & $I X^{e}-X^{e}$ \\
\hline 23. Orcet (63) & Zéra & $\overline{\mathrm{XIII}}{ }^{\mathrm{e}}$ \\
\hline 24. Pierrefitte sur Loire (оз) & Bois Vernillat (le) & $\mathrm{XII}^{\mathrm{e}}$ \\
\hline 25. Puy Guillaume (63) & Cabane (la) & $\mathrm{XIII}^{\mathrm{e}}$ \\
\hline 26. Saint-Prix (o3) & Jeanrais (les) & $\mathrm{VIIII}^{\mathrm{e}}-\mathrm{x}^{\mathrm{e}}$ \\
\hline 27. Toulon/Allier (о3) & Pré Des Dames (le) & $\mathrm{XIII}^{\mathrm{e}-\mathrm{XIV} \mathrm{V}^{\mathrm{e}}}$ \\
\hline 28. Trévol (о3) & Petite Perche (la) & $\mathrm{VIII}^{\mathrm{e}}-\mathrm{XIII}{ }^{\mathrm{e}}$ \\
\hline 29. Lezoux (63) & Colis (les) & $\mathrm{VII}^{\mathrm{e}}-\mathrm{VIII}^{\mathrm{e}} / \mathrm{XI}^{\mathrm{e}}$-XIV \\
\hline
\end{tabular}

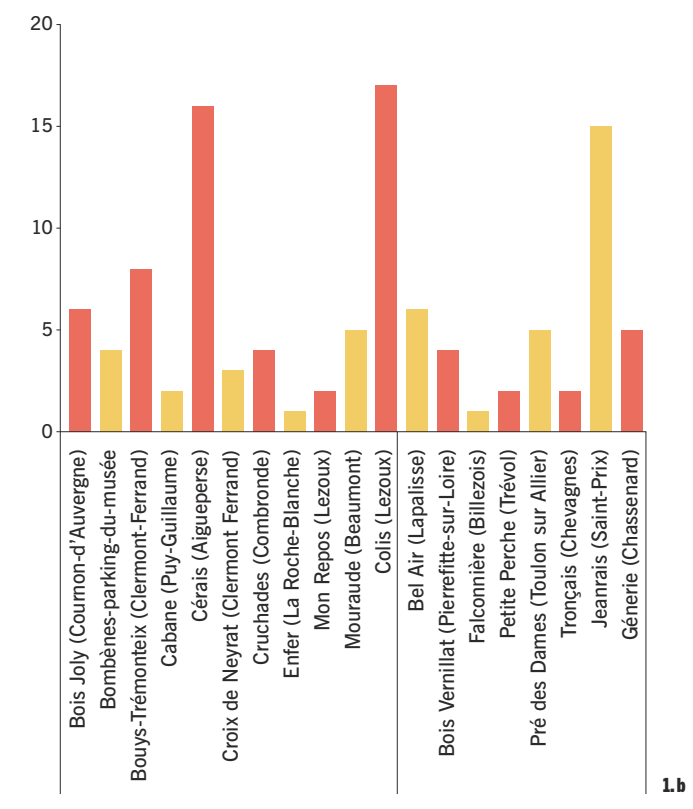


les édifices de plain-pied, les bâtiments excavés (avec le cas particulier des maisons adossées ou en encoche ${ }^{2}$ ) et enfin les édifices aériens qui sont exclusivement des annexes. Les bâtiments de plain-pied sont les plus courants dans notre corpus; si leur nombre est constant sur la période étudiée, le critère géographique paraît plus déterminant : durant le premier Moyen Âge, ils prédominent nettement dans l'Allier tandis qu'ils sont plus nombreux dans le Puy-de-Dôme au second Moyen Âge. Le nombre des bâtiments excavés, lui, va diminuant au fil du temps. Ce type de bâtiments est récurrent dans le Puy-de-Dôme, alors qu'il est très peu observé dans l'Allier. Mais s'agit-il de véritables habitations, de vides sanitaires ou bien encore de caves de bâtiments disparus comme c'est le cas pour les périodes antérieures, protohistoriques et romaines? Seule l'excavation du bâtiment 4 des Jeanrais a été interprétée comme un cellier, l'excavation étant réduite à une partie seulement de la surface d'un vaste bâtiment de plain-pied (Liégard, Fourvel, 2010). Dans les autres exemples, les profondeurs ne paraissent guère suffisantes pour correspondre à une cave ou à un vide sanitaire. Mais c'est surtout la présence de foyers aménagés sur de véritables sols d'occupation, avec du mobilier en position subhorizontale, qui amène à les interpréter comme des pièces d'habitation semi-enterrées.

L'enterrement de ces maisons résulte d'un choix puisque, si l'on écarte les maisons à encoche où le creusement est une facilité technique évidente, ces creusements ne sont pas liés à un type de substrat spécifique. Ils sont certes pratiqués dans des terrains stables aux Cérais, à la Petite Perche, et à Croix de Neyrat, mais également dans des terrasses alluviales meubles aux Cruchades et à la Mouraude, voire dans des sables à Lezoux, au Tronçais, et à Bois Joli. Sur le site de Croix de Neyrat, les constructions excavées coexistent avec celles de plain-pied, et l'habitat correspond sans équivoque à la partie enterrée, l'annexe réservée aux silos étant de plain-pied.

La profondeur de la part excavée peut aller de quelques centimètres (Bois Joli, les Cérais, la Cabane) à plus d'un mètre (Croix de Neyrat, la Petite Perche, Mon Repos, les Bombènes-parking du musée). On peut distinguer les structures entièrement excavées (37 cas) des structures adossées à une pente forte où la partie arrière est construite contre les parois d'un creusement (10 au total). Dans les cas les plus encaissés, les bords de l'excavation sont habillés de murets en pierre (Croix de Neyrat, les Cérais), de parois en bois (les Bombènes, Mon Repos) ou même de terre (Mon Repos, les Cérais). Les deux édifices les plus profonds sont construits indifféremment sur poteaux (Petite Perche avec 1,96 m), ou sur murs porteurs (les Cérais avec 1,90 m). Il est difficile d'y voir une réponse purement thermique puisque, dans la majorité des cas, la profondeur avoisine à peine le mètre.
Une typologie des édifices peut être élaborée à partir des trois types de structure porteuse qui coexistent sur la période : poteaux verticaux porteurs (poteaux poutres PP) ; murs porteurs ; structure mixte associant les deux. Le type le plus représenté est la maison sur poteaux poutres (55 exemples). Ces poteaux sont le plus souvent ancrés directement dans le sol dans un trou, plus ou moins profond, et souvent calés à l'aide de pierres. Un exemple, peut-être particulier puisqu'il s'agit d'un habitat seigneurial, a été retrouvé au Tronçais. Les négatifs des poteaux de la maison qui occupait l'enclos étaient tous circulaires. Or les 24 bois retrouvés étaient taillés sur section quadrangulaire. Les troncs ont été travaillés en laissant brute la partie enterrée circulaire, alors que la partie hors sol était quadrangulaire. Bien qu'impossible à détecter en fouille sauf lorsque le bois est préservé, cette technique est avérée à Husterknupp (Allemagne), à Schleswig et Haithabu pour les $\mathrm{X}^{\mathrm{e}}$-XII ${ }^{\mathrm{e}}$ siècle et à Pineuilh pour la même période (Chapelot, Fossier, 1980, p. 271; Vogel, 1994, p. 20). Les poteaux peuvent aussi se poser sur de maigres solins de pierre d'à peine une ou deux assises, voire sur des solins de bois (sablière) moins faciles à identifier. Une variante de cette technique consiste à faire reposer la charpente sur des poteaux qui sont pris dans des maçonneries de pierre ou de terre (11 cas). Faire reposer la charpente sur des murs porteurs de terre ou de pierre (43 cas) est plus rare mais devient la norme à partir de l'époque moderne.

\section{Les plans des bâtiments}

Les plans des bâtiments sont peu variés : ils sont en général rectangulaires (91 cas) ; le carré n'est attesté que pour des bâtiments annexes (11 occurrences) [ill. 2]. Leur surface, en revanche, va de $5 \mathrm{~m}^{2}$ à $100 \mathrm{~m}^{2}$ [ill. 3]. Les plans trapézoïdaux existent pour le premier Moyen Âge, mais il s'agit probablement plus d'une maladresse que d'une réelle volonté, car ils sont toujours irréguliers et sans angles droits. Comme le notait déjà JeanMarie Pesez, « la maison médiévale rurale ne connaît ni l'équerre ni le fil à plomb » (Pesez, 1986) et on pourrait rajouter « ni le compas ». Les bâtiments sur poteaux ont des plans plus divers, comprenant souvent plusieurs nefs, sans que l'on sache d'ailleurs s'il y a des partitions internes. Seuls les sites élitaires avérés, comme à la Mouraude, à Croix de Neyrat, au Tronçais ou encore au Colis possèdent des bâtiments à plusieurs pièces ; la majeure partie des édifices de notre corpus n'a qu'une seule pièce.

\section{Les matériaux et leur mise en ouvre}

Le détail des élévations reste le plus souvent assez hypothétique faute de données. L'association conjointe de plusieurs matériaux semble la méthode la plus répandue pour réaliser les murs : la pierre sert de soubassement pour une élévation en terre et bois. Les matériaux utilisés ne servent 
donc pas à différencier avec précision les fonctions des bâtiments.

La pierre, élément de différenciation régionale, se trouve dans les élévations de 17 maisons, toutes localisées dans le Puy-de-Dôme (la Mouraude, les Cérais, Bouys, Croix de Neyrat, les Bombènes). Dans la plupart des cas, il s'agit seulement de soubassements. Les avis divergent assez nettement concernant la restitution des élévations : seuls deux sites, peut-être trois, possèdent avec certitude des élévations en pierre dans la mesure où ces dernières constituaient la couche de démolition. Il ne s'agit que de pierres équarries, au mieux, jamais de pierres taillées, sauf cas ponctuels de remplois ; le liant est exclusivement composé de terre locale, sans mortier de chaux. À la Mouraude, l'utilisation de la pierre sur plus de $2 \mathrm{~m}$ de hauteur fait figure d'exception, tout comme à Bouys. Ailleurs, des élévations mixtes sont plus vraisemblables, comme aux Cérais et à Bois Joli. À Croix de Neyrat, les parties basses des maisons excavées sont construites en pierre. Mais il s'agit sans doute de murets supportant des élévations en terre, puisqu'ils s'associent à des poteaux verticaux. Sur le même site, le bâtiment 2 est un espace de plain-pied sur poteaux servant à abriter des silos ; l'arrière du bâtiment qui s'adosse à la partie habitation est construit en pierre alors que les trois autres parois sont édifiées sur poteaux.

Des élévations entièrement en bois ou en végétaux sont vraisemblables dans six cas. Des parois en roseaux ou végétaux sont évoquées à Bois Joli pour cinq des six bâtiments (MennessierJouannet et al., 2006). Avec plus de certitude, les parois du bâtiment 2 de Mon Repos étaient faites en planches d'au moins $2 \mathrm{~cm}$ d'épaisseur à en juger par la rubéfaction de l'ensemble des parois sur plusieurs centimètres, consécutive à un incendie. La présence de poteaux et de longs fragments ligneux contre les parois, au nord-est et nord-ouest, évoque un système d'armature en bois où les poteaux sont associés à des planches. Cette technique, connue notamment des archéologues nordiques, est aussi appelée stabbau, comme à Husterknupp (Chapelot, Fossier, 1980, p. 271) où les planches sont emboîtées dans des montants verticaux bouvetés. Ici, le système diffère un peu puisque la forme circulaire des bois retrouvés implique des planches horizontales. Peut-être s'agit-il d'une variante plus méridionale? Fouillée plus récemment par une équipe francoaméricaine, une maison excavée aux parois de planches a été découverte sur le site d'Yvérats à Compains [ill. 4] (Puy-de-Dôme) (Surmely, Franklin, 2016). Les comparaisons archéologiques manquent encore pour des raisons évidentes de conservation de ces éléments, mais aussi peut-être du fait de la prudence des archéologues : ainsi les élévations du bâtiment 4 des Bombènes, au mode constructif alliant solin et poteaux, auraient pu très bien pu être constituées de planches.
La technique précise utilisée pour construire les parois des bâtiments avec de la terre est difficile à déterminer. Si l'utilisation de la terre seule est souvent évoquée du fait de l'absence de vestiges, elle a été mise en évidence dans 20 cas. Sur ces sites ruraux où les études géomorphologiques tendent à montrer la faiblesse des apports sédimentaires, la présence de couches de terre recouvrant les vestiges indique, sans doute plus qu'on ne le croit, l'usage de terre pour les élévations. Différentes techniques existent, allant de la simple terre humide malaxée (bauge), à celle de la brique de terre crue (adobe), en passant par le pisé qui suppose l'existence d'un coffrage, mais aussi le torchis sur clayonnage de bois ou pan de bois (Perello, 2015). Ces différentes techniques sont toutes vraisemblables et parfois attestées au hasard de la conservation des éléments.

Le torchis de terre sur armature de bois est manifeste dans 43 cas. Ce procédé s'observe très bien au Tronçais grâce à l'incendie de la maison : un épais torchis de terre $(5 \mathrm{~cm})$ est posé sur un perchis de bois, fait de barreaux régulièrement espacés et enfoncés sur une sablière en bois. En Franche-Comté, sur le site de Petit Noir, aux XIII ${ }^{\mathrm{e}-}$ $\mathrm{XIV}^{\mathrm{e}}$ siècles, les torchis, d'une épaisseur moyenne de $4 \mathrm{~cm}$, sont appliqués sur des structures à pan de bois et recouverts d'un enduit de chaux (Méloche, 2015b ; Gauthier F. 2019). Le torchis sur clayonnage constitue l'hypothèse la plus plausible pour 34 bâtiments de l'Allier (Bois Vernillat, le Pré des Dames, les Jeanrais, Bel Air et la Générie). Cette technique de parois en terre crue est parfaitement adaptée à la Sologne bourbonnaise (substrat composé de sables et d'argiles, peu de pierres mais abondance de bois de construction). Au Xv siècle, l'obligation « de refaire les bas torchis tous les ans dans certains baux renseigne sur la fragilité des matériaux et sur l'absence des solins en pierre qui isolent de l'humidité du sol ». En revanche, dans le Puy-de-Dôme, seuls 7 bâtiments semblent illustrer cette technique.

L'utilisation de briques de terre crue a été mise en évidence uniquement pour la maison de la Petite Perche qui conservait des mottes de terre façonnées, associées à des morceaux de torchis sur clayonnages. La brique d'adobe, présente dans la région de Lezoux, est encore visible sur des constructions modernes dans la bourgade. On la trouve aussi dans les couches supérieures de la motte castrale de Lempty (Puy-de-Dôme).

Quant à la technique de la bauge, elle n'est manifeste que pour une cloison en terre encore partiellement en élévation, découverte dans le bâtiment excavé F96 aux Cérais. Cette cloison, construite directement sur le fond du creusement, s'appuyait contre la paroi de marne. Il s'agissait d'un mélange compact de marne, de cailloutis calcaire tiré du substrat et sans doute de fibres végétales. L'absence de traces de banches, ainsi que la datation haute $\left(\mathrm{x}^{\mathrm{e}}\right.$ siècle), laissent plutôt présager une technique proche de la bauge où la terre est 
2. Au cours de la période, le nombre de bâtiments excavés évolue assez

nettement. L'opposition entre

les deux départements est

forte au premier Moyen Âge

( 2 bâtiments excavés dans

I'Allier ; 26 dans le Puy-

de-Dôme) et se prolonge

au second Moyen Âge

( 2 bâtiments excavés dans

l'Allier ; 17 dans le Puy-

de-Dôme).

Lorsque les entrées ont pu

être repérées, elles sont

majoritairement orientées

à l'est (18 cas) ou au sud

(11 cas), ce qui est le plus

logique d'un point de vue

climatique. Les autres

orientations (7 à l'ouest et

6 au nord) semblent le fait

de contingences particulières

(voies, autres bâtiments...).
VI $^{\mathrm{e}}$-VIIII ${ }^{\mathrm{e}}$ siècle

L'Enfer (La Roche Blanche)

BÂTIMENT B

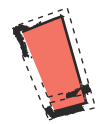

Bois Joli (Cournon) viI ${ }^{\mathrm{e}}$ siècle

BÂT.

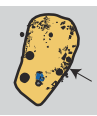

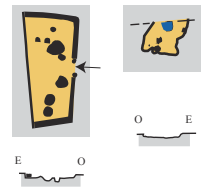

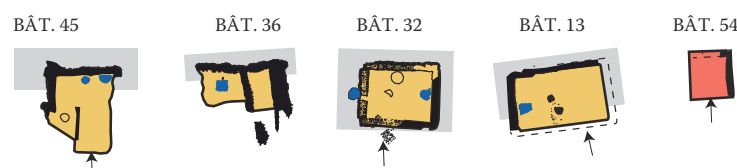

Les Colis (Lezoux)

BAT 25

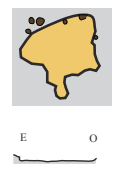

Bouys

(Clermont-Ferrand)

VIII $^{\mathrm{e}}-\mathrm{X}^{\mathrm{e}}$ siècle
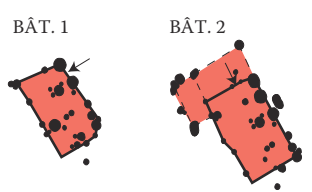

BÂT. 3
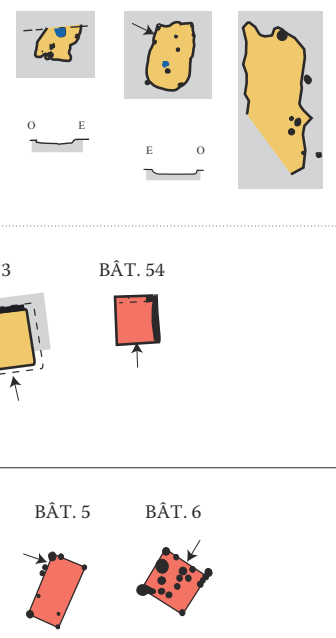

La Petite

Perche

(Trévol)
F 93

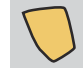

Bel-Air (Lapalisse) $\mathrm{IX}^{\mathrm{e}}$ - $\mathrm{X}^{\mathrm{e}}$ siècle
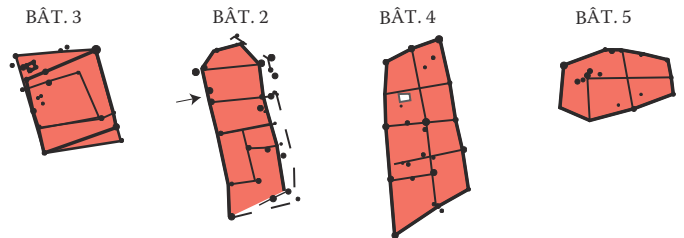

BÂT. 6

BÂT. 7

La Génerie
IX-XI
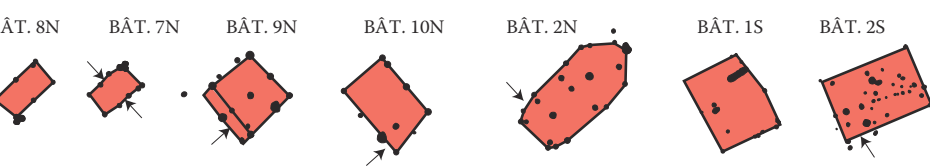

BÂT. $1 \mathrm{~N}$ BÂT. 3N BÂT. $4 \mathrm{~N}$

BÂT. $5 \mathrm{~N}$

BÂT. 6N

BÂT. $11 \mathrm{~N}$

\section{$\square<\Delta<\Delta$}

Les Jeanrais
Croix-Neyrat

(Clermont-Ferrand)

$\mathbf{x}^{\mathrm{e}}-\mathbf{X I}{ }^{\mathrm{e}}$ siècle

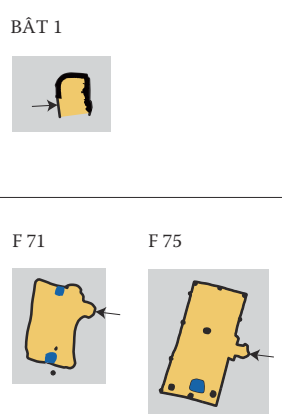

Bouys

(Clermont-Ferrand) $\mathbf{X}^{\mathrm{e}}-\mathbf{X I}^{\mathrm{e}}$ siècle

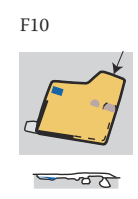

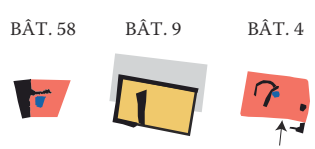

7.
Les Cérais (Aigueperse) $\mathrm{XI}^{\mathrm{e}}$ siècle
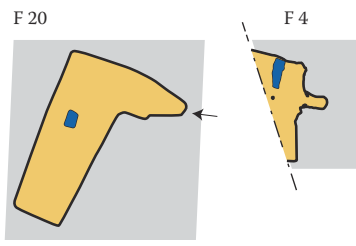
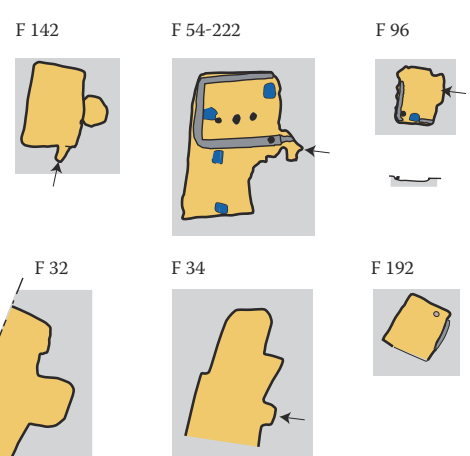
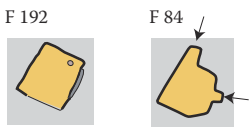

$\square$
Le Parking du musée (Lezoux)

XI-XII ${ }^{\mathrm{e}}$ siècle $\square$ bâtiment excavé

$\square$ bâtiment de plain pied
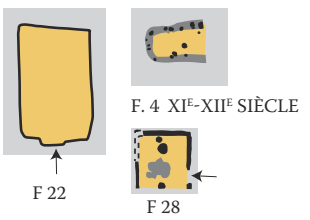

Mon Repos

(Lezoux)

$\mathbf{X I}^{\mathrm{e}}$-XII ${ }^{\mathrm{e}}$ siècle

.

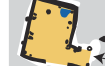

BÂTIMENT 1-F528

(A,1963)
Croix-Neyrat

(Clermont-Ferrand)

XI-XII ${ }^{\mathrm{e}}$ siècle BÂT 3

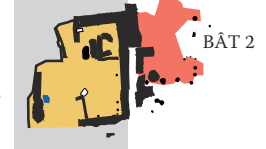



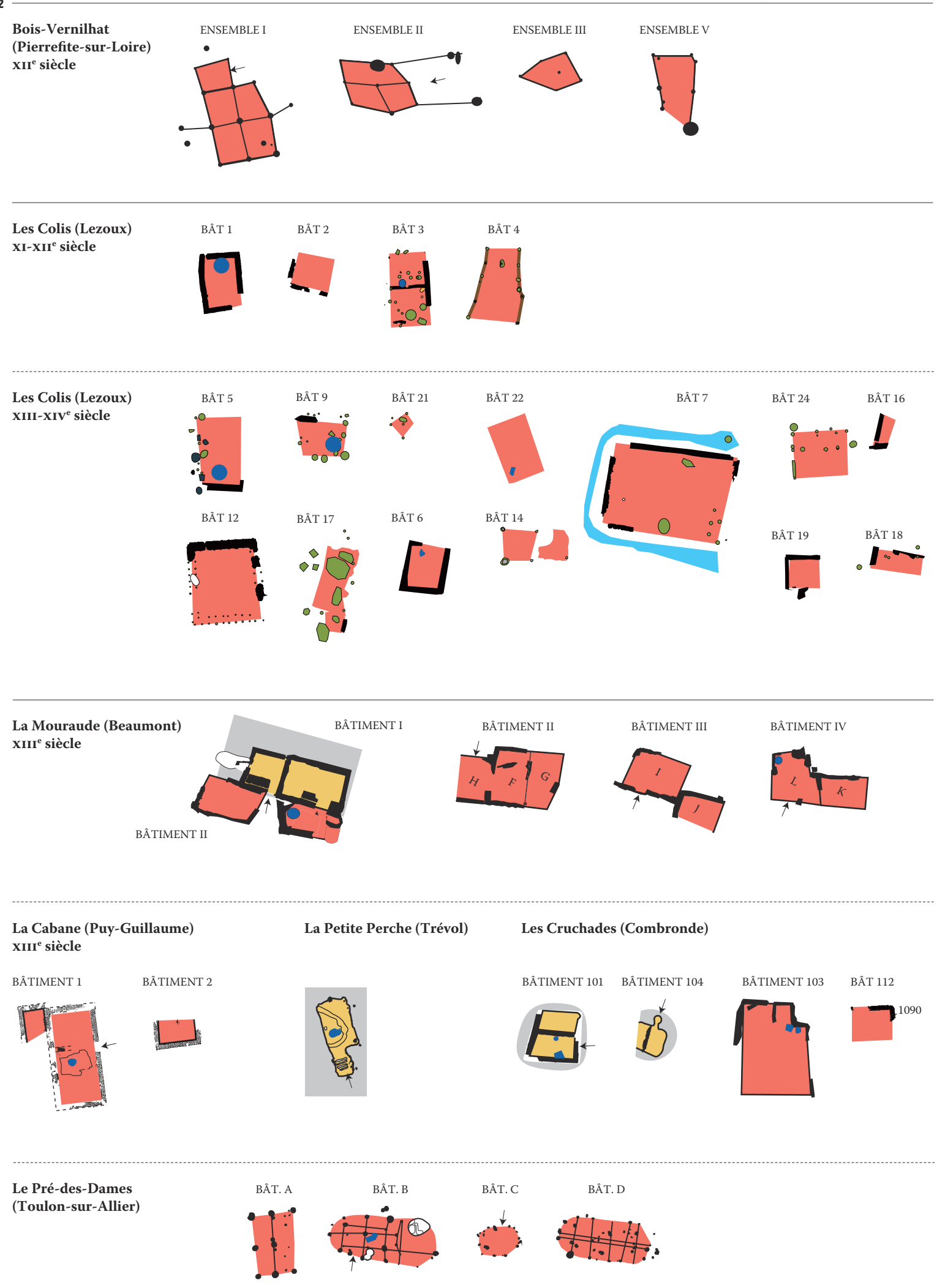

Falconnière (Billezois)

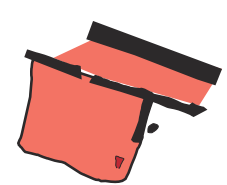

Le Tronçais (Chevagne) 1359-1360
Croix-Neyrat

(Clermont-Ferrand)

$\mathrm{XIV}-\mathrm{XV}^{\mathrm{e}}$ siècle

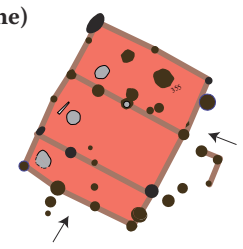

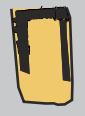

BÂT 4 


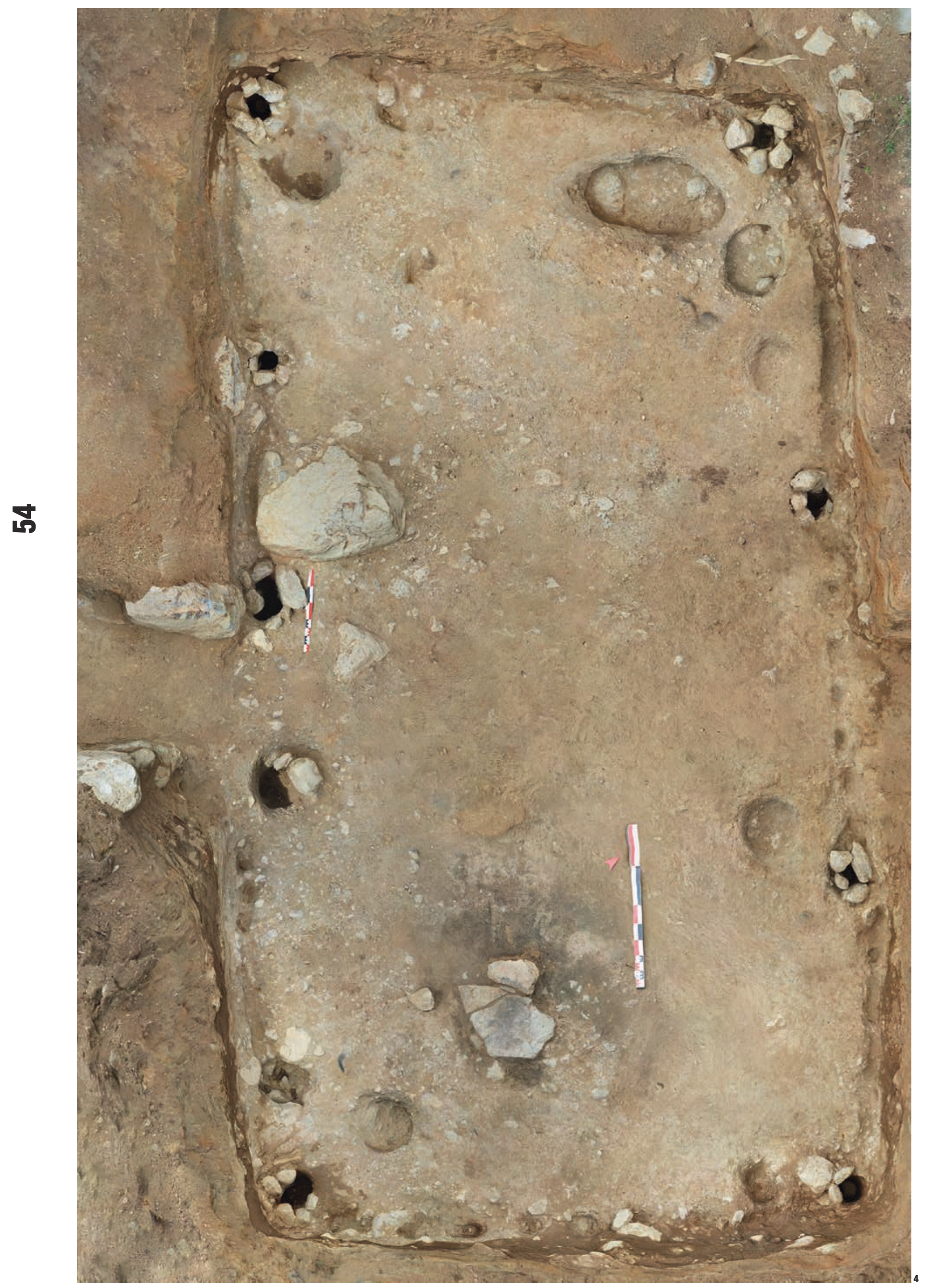




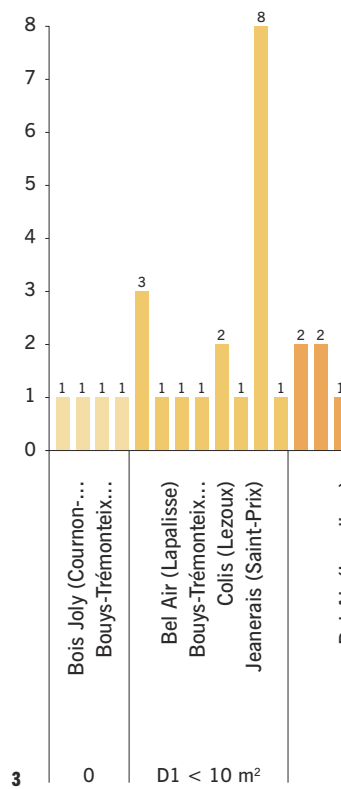

$\mathrm{D} 1<10 \mathrm{~m}^{2}$

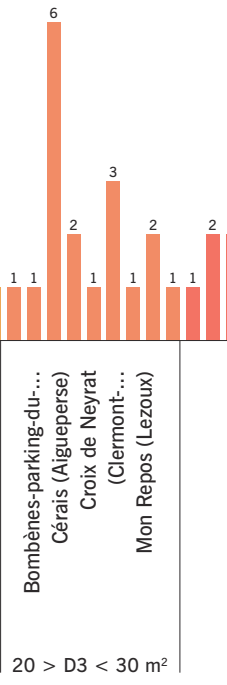

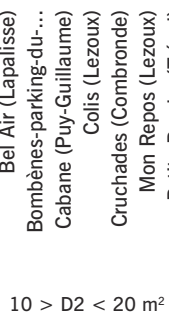

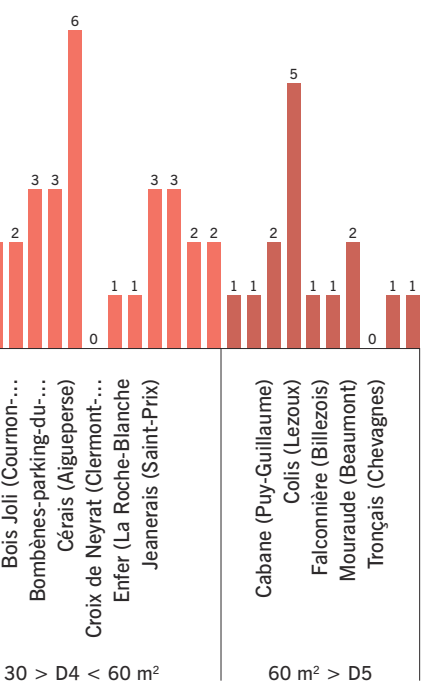

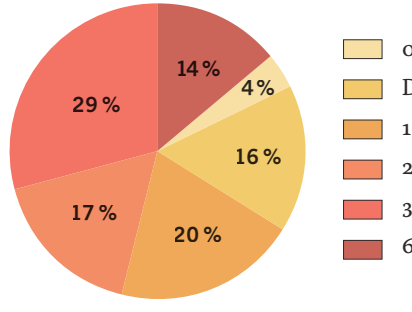

$\mathrm{D}_{1}<10 \mathrm{~m}^{2}$

$10>\mathrm{D}_{2}<20 \mathrm{~m}^{2}$

$20>\mathrm{D}_{3}<30 \mathrm{~m}^{2}$

$30>\mathrm{D}_{4}<60 \mathrm{~m}^{2}$

$60 \mathrm{~m}^{2}>\mathrm{D}_{5}$
3. Les surfaces des bâtiments présentent un large éventail que l'on peut répartir en cinq groupes. 62 édifices, soit la majorité, ne comprennent qu'une pièce unique, inférieure à $30 \mathrm{~m}^{2}$. Notons qu'aujourd'hui, la surface minimale pour une maison est de $30 \mathrm{~m}^{2}$.

4. Maison excavée aux parois de planches des Yvérats, à Compains, dans le Puyde-Dôme.
3. Voir les travaux

d'Olivier Aurenche et

Claire-Anne de Chazelles et Archéopages 42

Construire en terre crue,

2015.

4. La terre qui va

constituer les murs

est versée dans un

coffrage en planches

en bois disposées

horizontalement. Une

fois déposée entre les

planches, la terre est

damée depuis l'intérieur

du coffrage.

5. Cette particularité

peu fréquente est visible

sur des bâtiments

modernes marocains

(communication orale

Abdallah Fili). façonnée à la main sans coffrage. Comme pour la Petite Perche, les élévations de ce bâtiment étaient assez composites. Cette technique pourrait avoir été employée pour l'ensemble du hameau des Cérais.

Force est de reconnaître que le terme de pisé est utilisé de manière assez aléatoire voire contradictoire dans la littérature archéologique, malgré des tentatives d'harmonisation terminologiques ${ }^{3}$. Bien qu'il s'agisse d'un pléonasme, nous employons à dessein le terme « pisé banché » pour bien distinguer cette technique particulière de l'usage simple de la terre crue où la technique précise n'a pas été identifiée. L'étude auvergnate atteste dans 13 cas des élévations en pisé banché (les Colis, Mon Repos, la Cabane) ce qui constitue une curiosité par rapport au reste de la France (Chazelles, Thernot, 2015). Pourtant en Auvergne, l'utilisation du pisé pour une élévation médiévale est notée dès 1963 dans le bâtiment 1-F528 de la fouille de Mon Repos à Lezoux. Les fouilleurs anglais ont formellement identifié cette mise en œuvre, tout en notant bien l'aspect composite des élévations : «... murs en pisé dont le coin était une maçonnerie de pierres et de tuiles consolidées à l'argile » (Frere, Hartley, 1966). Cette découverte est d'ailleurs assez exceptionnelle pour être mentionnée par Jean Chapelot et Robert Fossier dans leur ouvrage de réference (Chapelot, Fossier, 1980, p. 264), faisant de cette dernière un des jalons le plus septentrional pour la redécouverte de cette technique durant le Moyen Âge (Chazelles, Thernot, 2015). En effet, malgré les réserves parfois formulées, dues aux confusions entre pisé et bauge (Chazelles, Guyonnet, 2007), la reprise et l'achèvement de la fouille en 2011 ont confirmé le recours au pisé au moins pour le mur M1 (Bet, 2015). Cette technique a depuis été à nouveau identifiée à Lezoux aux Colis pour la même période chronologique. Sur ce dernier site, pour une période comprise entre la fin du XI $I^{e}$ et le début du XIV ${ }^{e}$ siècle, on compte au moins neuf bâtiments construits ainsi.

L'identification repose sur la présence de négatifs de piquets pour caler les banches sur au moins trois édifices (le bâtiment 7 , le bâtiment 9 et surtout l'exceptionnel bâtiment 12). Ces vestiges prouvent ici l'utilisation de la technique du «pisé banché » au sens strict du terme ${ }^{4}$. L'écartement entre les banches est ici suffisant (entre 0,80 et $0,96 \mathrm{~m}$ ). Les piquets retrouvés correspondent à des poteaux verticaux comme l'attestent les profils des négatifs, il ne semble donc pas y avoir de contreventement comme dans la pratique moderne. Il n'y a sans doute pas non plus de " clefs », ces pièces de bois posées en travers du mur montant, pour soutenir au fur et à mesure les banches. L'écartement est simplement assuré par des entretoises et des tirants de corde attachés aux poteaux. Mais, outre ces négatifs de piquets, la typologie diffère pour les solins du bâtiment 1 . Sans doute un peu plus précoces puisque construits au $\mathrm{XII}^{\mathrm{e}}$ siècle, seuls les parements du solin [ill. 5] sont construits avec des tessons ou des galets, afin d'assurer la fermeture des banches et d'empêcher la terre de couler au moment du damage ${ }^{\mathbf{5}}$. Le pisé banché est également présent sur le site de la Cabane (2o km au nord de Lezoux), à la même époque, ainsi qu’à Rossignol (commune de Changy, Loire, contiguë à la montagne bourbonnaise) où ont été fouillés quatre bâtiments du XIV siècle (Gaime, Franc, Thévenin et al., 2005).

Ces constructions médiévales en pisé banché préfigurent la technique moderne mais diffèrent dans la mise en œuvre. Contrairement aux maisons modernes, le solin médiéval n'existe pas toujours 

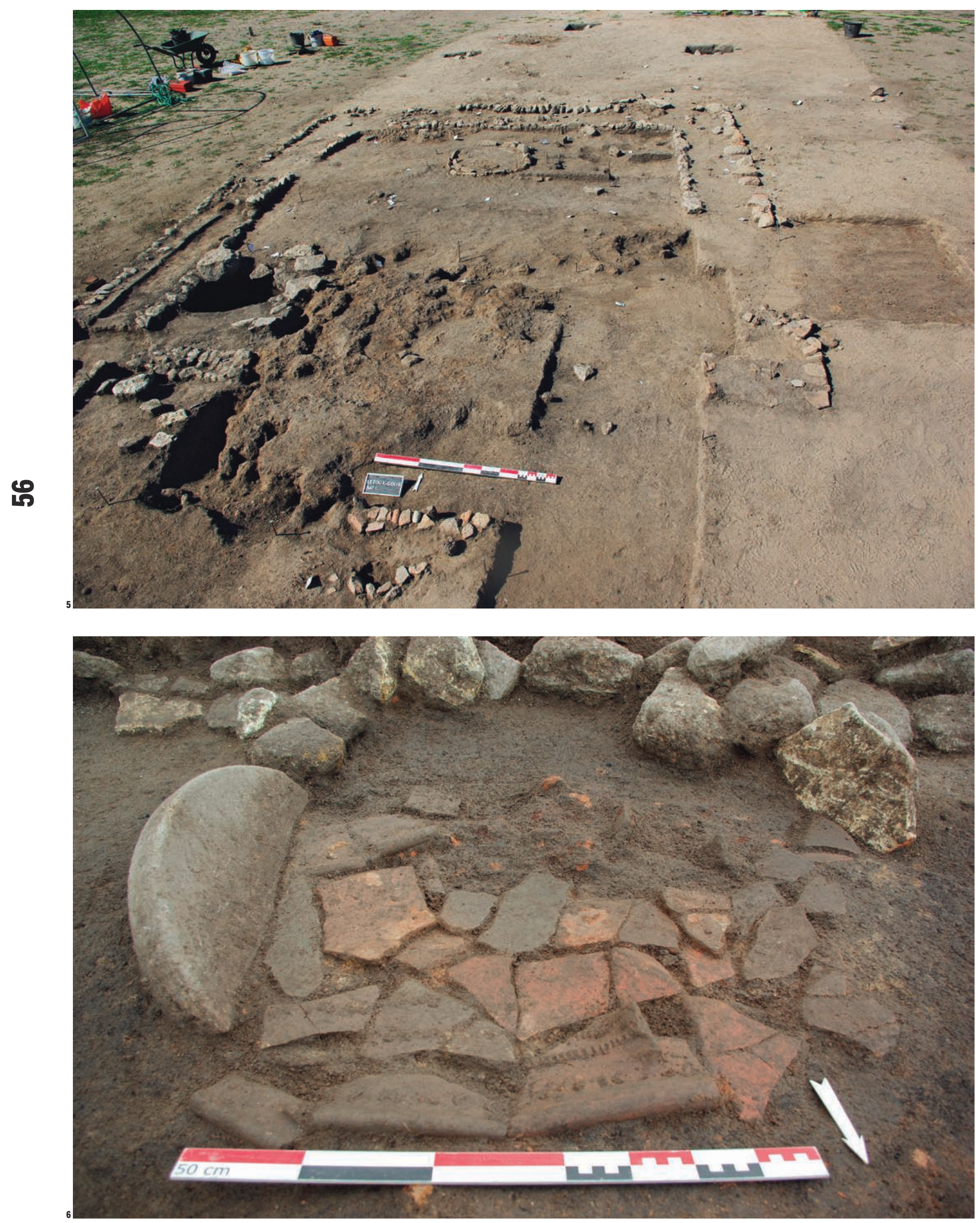
et ne consiste au mieux qu'en une assise de pierres (la Cabane, Mon Repos, les Colis). De même, une fine couche de mortier de chaux sépare souvent les banches modernes, attestant une évolution dans la technique. Ces différents sites permettent d'esquisser un schéma évolutif. Encore inconnue aux Bombènes-parking-du-musée, entre le $\mathrm{XI}^{\mathrm{e}}$ et le $\mathrm{XII}^{\mathrm{e}}$ siècle, le pisé banché apparaît timidement sur un seul mur du bâtiment 1-F528 de Mon Repos. Ce bâtiment n'utilise pas uniquement le pisé mais une architecture composite, avec une structure de type poteau porteur. On peut se demander si ce bâtiment, légèrement antérieur aux maisons découvertes aux Colis (Gaime et al., 2016) ou à la Cabane, ne constitue pas un jalon technique composite avant la généralisation du pisé strict tel qu'il est attesté localement au XII ${ }^{\mathrm{e}}$ et surtout au $\mathrm{XIII}^{\mathrm{e}}$ siècle. Le mur de pisé est celui où vient s'adosser le foyer, remplaçant même ici le poteau soutenant la faîtière. Par crainte d'incendie, le pisé serait venu supplanter une élévation partiellement en bois. Aux Colis, les deux états du bâtiment 3 laissent envisager une évolution similaire, allant de l'usage du poteau planté (état 1 , fin XII ${ }^{\mathrm{e}}$ siècle) vers le pisé (état 2, XIII ${ }^{\mathrm{e}}$ siècle). Les données auvergnates nuancent donc la proposition faite pour la France du sud où les données disponibles attestent l'apparition du pisé au plus tôt au XII ${ }^{\mathrm{e}}$ siècle (Chazelles, Thernot, 2015).

\section{La toiture et la couverture}

Dans la documentation archéologique, la toiture est restituée en se fondant sur les largeurs des constructions, la répartition des appuis verticaux et les matériaux utilisés pour les élévations. Les toitures à deux pans prédominent largement dans les restitutions. C'est le mode quasi exclusif envisagé aussi bien pour les constructions sur murs porteurs que pour les structures sur poteaux. Dans les cas de poteaux porteurs, des calages axiaux impliquent une faîtière et une toiture à deux pans. C'est le cas à la Petite Perche (bâtiment 2), à Mon Repos, à la Générie (bâtiment 1), à Bel Air (bâtiments 1 et 2) et aux Jeanrais (bâtiment 2), aux Cérais ( $\mathrm{F}_{71}$ ), au Tronçais et à Croix de Neyrat (bâtiment 3). Technique un peu plus élaborée, des pignons en croupe sont probables pour les constructions aux plans arrondis ou triangulaires (les Jeanrais, la Générie, Bois Joli, la Petite Perche). Enfin, le plat supérieur de la sablière haute retrouvée au Tronçais recevait la tombée des chevrons. La présence de l'assemblage en long à enture oblique montre que cette pièce se positionnait sur une des longueurs du bâtiment. Elle possédait une entaille à mi-bois qui situe l'emboîtement de l'entrait d'une ferme. Au même niveau sur le plat inférieur, une grande mortaise situe l'encastrement du tenon d'un poteau porteur. Il s'agit là d'une construction à ossature bois (Gaime, Gauthier, Mille et al., 2011).

L'absence des restes archéologiques de la couverture plaide en faveur de l'utilisation de matériaux périssables. Les sites de Falconnière et du Tronçais, datés du XIV ${ }^{\mathrm{e}}$ siècle, ont documenté l'utilisation de tuiles en bois, dont on ignorait l'existence jusqu'alors dans la région. Ces bardeaux provenant de sites élitaires (un château et un moulin), on peut se demander si leur usage doit être considéré comme un marqueur social dans le Bourbonnais. La fixation par cheville en bois ne laissant jamais de traces, nous manquons encore d'éléments mais cette technique est bien attestée ailleurs (Méloche, 2015a) à la même époque. Il est possible que ce type de couverture soit beaucoup plus répandu qu'il n'y paraît. C'est ici que les restitutions s'opposent : entre la chaumière recouverte de branchage et la toiture en tavaillon. Comme souvent, la vérité est sans doute entre les deux. Quoi qu'il en soit, ces découvertes remettent en question l'idée d'une maison paysanne systématiquement couverte en chaume, peut-être un peu trop véhiculée par les cartes postales du début du $\mathrm{XX}^{\mathrm{e}}$ siècle. Des couvertures en roseau ou en jonc paraissent probables pour les maisons de Bois Joli, en raison de la proximité d'une roselière, de la légèreté des charpentes envisagées, ainsi que de l'emplacement central des foyers. Comme ailleurs en France, les tuiles de terre cuite ou d'ardoise sont totalement absentes des campagnes auvergnates au Moyen Âge.

\section{Les aménagements intérieurs}

De manière générale, c'est la simplicité qui prévaut. L'intérieur devait être assez sombre, avec peu de fenêtres (le verre est totalement absent) et l'éclairage principal devait provenir du foyer. Plus curieux est l'absence de lampe à huile ${ }^{\mathbf{6}}$. Stockages, sols et foyers constituent des critères de définition plus généralement documentés en fouille. On compte plusieurs exemples de structures de stockage directement au sein de la maison : simple fosse creusée dans le substrat (Bois Joli, Croix de Neyrat) ; coffre de bois semienterré à l'intérieur d'un bâtiment (à la Générie, il contenait des grains de seigle et du raisin); creusement quadrangulaire à parois de pierre dans le sol, recouvert d'un couvercle de bois.

Pour des raisons taphonomiques, la plupart des sols de maisons conservés l'ont été dans des structures excavées. Il s'agit généralement du substrat taillé, qu'il soit dur (calcaire) ou meuble (sable, terre, argile...), mis à profit sans traitement spécifique, sauf peut-être des recouvrements de végétaux qui, à la longue, forment les litages successifs si caractéristiques des couches d'occupations. Le devant du bâtiment de Falconnière possédait une surface aménagée par des morceaux de tegulae, un seuil en dalles de pierre existait aux Cérais, enfin le sol du bâtiment B de l'Enfer était fait pour partie de terre battue, pour partie de mortier de chaux, suggérant peut-être une partition de la pièce. Les sols des bâtiments de la Mouraude étaient plus élaborés : le sol initial du bâtiment 1 , le plus vaste, entièrement construit en

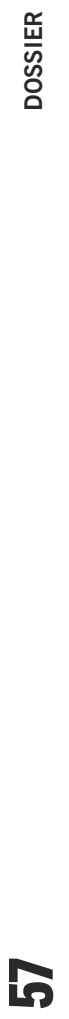

ก 
pierre, était constitué d'un pavement rudimentaire de pierres plates plus ou moins jointives.

Sept bâtiments de l'Allier et 4,2 du Puy-deDôme sont équipés de foyers, qui se trouvent le plus souvent à même le sol et parfois dans un léger creusement : 25 dans des bâtiments excavés, 7 dans des édifices adossés, et seulement 17 dans les bâtiments de plain-pied. Ces derniers, majoritairement en bois, disposent rarement d'un foyer ouvert. La norme, d'un seul foyer par édifice, connaît des exceptions aux Cruchades et aux Cérais, où certains édifices en possèdent deux ; la maison forte du Tronçais en a trois. Le fait plus notable est que 68 bâtiments n'en possèdent pas, ce qui impliquerait, si l'on se référait à ce critère de définition, que moins de la moitié des bâtiments du corpus correspondent à des maisons d'habitation. Le foyer de la maison rurale médiévale est généralement un foyer ouvert, aux formes circulaires ou rectangulaires. Les soles peuvent être aménagées à l'aide de dalles de pierre locale, d'éléments de céramique ou de morceaux de tegulae. Elles sont parfois circonscrites par des pierres sur chant dessinant une sorte de couronne, par des céramiques ou des pierres [ill. 6] ; il peut aussi s'agir simplement d'une sole d'argile. À Bouys, il semble qu'un soin particulier ait été pris pour les soles, construites dans trois cas avec un matériau réfractaire local (le téphra volcanique) enrobé dans de l'argile. Les recharges successives de sédiments pour l'entretien des sols de circulation obligent à surélever le foyer pour éviter qu'il ne soit en contrebas. Cas unique dans le corpus, une plaque foyère a été identifiée dans le bâtiment 2 de Mon Repos. Quelques pierres sur chant évoquent des chenets rudimentaires dans le bâtiment 4 à Bouys. Sans spécificité apparente, cette seule source de feu possède sans doute des fonctions multiples. La fonction culinaire de ces foyers situés à l'intérieur d'un habitat ${ }^{7}$ a été quelques fois repérée grâce aux traces de mises au feu visibles sur les céramiques retrouvées (Tronçais, Croix de Neyrat). Aux Colis, la présence de quelques scories et battitures autour du foyer laisse penser qu'il était ponctuellement le lieu d'activités artisanales.

L'aménagement des foyers correspond donc au strict nécessaire et, la plupart du temps, aucun système d'évacuation n'est envisagé, ce qui impliquerait une présence continue de fumée dans l'habitation. Toutefois, la ténuité des traces ne prouve pas l'absence d'aménagements, plus que vraisemblables à la Mouraude, à Croix de Neyrat, au Pré des Dames, à Mon Repos, aux Cérais et au Tronçais. Dans les cas des foyers plaqués contre un mur au milieu du pignon, il est plausible d'envisager une élévation en matériaux non inflammables (pierre ou pisé). Deux exemples suggèrent une évacuation de la fumée par un point haut, et sans doute par un conduit au vu du décalage ménagé entre le foyer et la poutre faîtière. Ainsi aux Cérais, pour le bâtiment F75, deux poteaux encadrent un foyer décentré pour éviter la poutre faîtière, un espace semble matérialiser le négatif d'une paroi isolante, venant doubler le pignon et soutenir un système d'évacuation des fumées. À la Mouraude, le foyer de la pièce E est une sole circulaire construite dans l'angle. En avant du foyer, les négatifs de deux poteaux et un alignement de pierres sur le sol de la pièce, associés à un linteau monolithe à l'arrière, évoquent fortement une hotte. Au Tronçais, trois foyers sont disposés chacun au sein d'une travée, contre le pignon oriental et à une distance de plus d'un mètre de la paroi. Le deuxième, qui occupe la plus grande travée, est séparé de la paroi par une trace linéaire qui évoque l'existence d'une structure d'évacuation et de protection. Enfin, aux Colis, le foyer circulaire du bâtiment 3, délimité par des pierres et un fragment de meule, s'implante en partie dans la paroi elle-même, suggérant l'existence d'un conduit de cheminée.

L'ustensile complémentaire du foyer est le couvre-feu ${ }^{\mathbf{8}}$. Cet objet en céramique a sans doute diverses utilités : éviter les incendies lorsque le feu n'est pas surveillé, la nuit notamment, empêcher que le feu ne s'éteigne trop vite, permettre de le ranimer le matin sans avoir à le rallumer. Mais il peut aussi transformer le foyer en une sorte de «four » comme le suggère parfois la présence de trous réguliers dans la paroi, permettant un apport d'air tout en bénéficiant du caractère réfractaire de la terre cuite. Comme il était inconnu sur nos sites jusqu'en 2001, on le pensait réservé aux habitats seigneuriaux. Or il s'agit bien d'un équipement de la maison paysanne auvergnate médiévale, repéré dès le $\mathrm{x}^{\mathrm{e}}$ siècle dans le mobilier du bâtiment F75 des Cérais, et présent sur les sites du Pré des Dames, de Mon Repos, des Cérais, de Falconnière, du Tronçais, de Bouys, de la Mouraude et des Colis.

Avec une certaine ironie, le temps de la recherche archéologique auvergnate appartient à ce temps long qu'appelait de ses vœux Fernand Braudel, puisque 48 années se sont écoulées entre la première fouille d'une maison médiévale de Mon Repos à Lezoux, dite alors de « sauvetage programmé », réalisée en 1963, sous la codirection de Sheppard Frere et Brian Hartley, et son achèvement, en 2011, comme « fouille préventive » sous la direction de Philippe Bet (Bet, 2015). Entretemps, une deuxième maison médiévale située dans le terrain voisin a été fouillée (MennessierJouannet, 1993). Les données issues de la première fouille sont reprises dans la célèbre synthèse sur le village et la maison au Moyen Âge évoquant alors la technique du pisé pour « les fonds de cabane à Lezoux du XIII ${ }^{\mathrm{e}}$ s. » (Chapelot, Fossier, 1980, p. 264). Aujourd'hui, la datation de ces édifices a été reculée à la fin du $\mathrm{XI}^{\mathrm{e}}$ ou au début du XII ${ }^{\mathrm{e}}$ siècle (Bet, Gaime, Horry, à paraître) et la mise en œuvre du pisé confirmée.

Grâce à l'archéologie, notamment préventive, il est possible aujourd'hui d'entrevoir la maison
7. Aucun four de type communautaire n'a été retrouvé pour l'instant. 8. De forme circulaire, plus ou moins décoré, cet objet a été observé pour la première fois en Hongrie (cité dans Pesez, 1986, p. 83). 
rurale médiévale auvergnate au moins dans deux régions. Malgré des lacunes certaines, les données semblent récurrentes. Ainsi les plans sont toujours proches du rectangle, avec au début de la période étudiée quelques variations dans les pignons pour les bâtiments sur poteaux. Le plan va ensuite se fixer durant le second Moyen Âge. La mixité homme-animal attestée dans certaines régions par la fouille, relayée par l'ethnographie (Conte, Fau, Hautefeuille, 2010), ne semble pas exister en Auvergne sauf dans les habitats de moyennes montagnes non pris en compte ici. Pendant toute la période étudiée, la maison n'a qu'une seule pièce, dont la surface moyenne oscille entre 20 et $30 \mathrm{~m}^{2}$, sauf lorsqu'il s'agit de sites élitaires et non de simples habitats paysans.

Dans le monde rural médiéval, on construit avec ce que l'on a au plus près, ce qui induit certaines différences régionales. Là, les différences entre le Bourbonnais et la Limagne apparaissent. Grâce aux forêts de la Sologne bourbonnaise, le recours au bois est plus fréquent que pour les constructions de la Limagne d'Auvergne. Quant à la terre, elle est toujours utilisée crue ; en Sologne bourbonnaise, elle recouvre les clayonnages et forme les torchis, alors qu'en Limagne ce sont les murs eux-mêmes qui sont en terre crue. Pour les couvertures, les deux régions emploient les végétaux. De manière schématique, la maison paysanne bourbonnaise est plutôt construite à pans de bois, avec une structure porteuse sur poteaux, de plain-pied, et possède des élévations en torchis, avec une toiture végétale ou en bardeaux. En Basse-Auvergne, la maison paysanne est plutôt excavée avec des murs en pierre ou en pisé, porteurs ou non, et une toiture végétale.

Force est de constater qu'il y a peu d'évolutions architecturales sur la période étudiée, sauf peut-être une tendance à construire des plans plus réguliers, le progrès des charpenteries repéré sur les édifices religieux se répercutant peut-être peu à peu dans la construction vernaculaire, avec l'émergence de corps de métiers spécialisés. L'apparition assez timide, aux alentours du XII siècle, du pisé banché, très localisé dans la région de Lezoux, devance aussi ce que l'on croyait jusqu'à présent. Dans les campagnes, le passage à la construction de pierres et aux couvertures de tuiles ne semble pas s'opérer avant l'époque moderne. Il n'y a donc pas d'argument typochronologique précis et, du point de vue formel, dater un édifice sans le recours au mobilier qui y a été retrouvé est plus que difficile.
Alfonso G. et al., 1998, Aigueperse (Puy-de-Dôme), les Cérais, habitat rural du haut Moyen Âge. Nécropole de l'Âge du Fer, rapport d'opération, Afan-SRA Auvergne, 3 vol.

Baucheron F., 2007, Lezoux (Puy-de-Dôme), les Bombènes, rapport d'opération, Inrap-SRA Auvergne, $138 \mathrm{p}$.

BAUCherON F. et al., 1999, Toulon-sur-Allier, Lieu-dit: Le-Pré-des-Dames, DFS, Afan-SRA Auvergne, $152 \mathrm{p}$.

BAucheron F. et al., 2005, La-Roche-Blanche-Orcet (Puy-de-Dôme), l'Enfer, rapport d'opération, InrapSRA Auvergne, 2 vol.

Bet P. et al., 2015, Lezoux (Puy-de-Dôme). Rue Pasteur, la fouille de la Maison de retraite Mon Repos 2011, ateliers antiques et habitat médiéval, rapport d'opération, Inrap-SRA Auvergne, $250 \mathrm{p}$.

Bet P., GAIME S., Horry A., à paraître, Les Bombènesparking du musée (Lezoux, Puy-De-Dôme).

Chapelot J., Fossier R., 1980, Le village et la maison au Moyen Âge, Paris, Hachette, « Bibliothèque d'Archéologie ", $358 \mathrm{p}$.

Chazelles C.-A. DE, Guyonnet F., « La construction en pisé du Languedoc-Roussillon et de la Provence, du Moyen Âge à l'époque moderne (XIII $-\mathrm{XIX}^{\mathrm{e}} \mathrm{s}$.) », in Guillaud H., Chazelles C.-A. De, Klein A. (dir.), Échanges transdisciplinaires sur les constructions en terre crue, 2 : la terre massive, Mai 2005, Villefontaine (Isère), France, Éditions de l'Espérou, École Nationale Supérieure d'Architecture de Montpellier, 1, p. 109-139.

Chazelles C.-A. De, Thernot R., 2015, " La construction en terre crue coffrée et damée dans le sud de la France au Moyen Âge. Transfert des techniques ou migration des techniciens? », in Richarté C., Gayraud R.-P., Poisson J.-M. (dir.), Héritages arabo-islamiques dans l'Europe méditerranéenne, Actes du colloque de Marseille, 11-14 septembre 2013, Paris, La Découverte, p. $253-267$.

Chuniaud K. et al., 2013, Clermont-Ferrand (Puy-deDôme), ZAC de Trémonteix. D'un domaine l'autre, rapport d'opération, Inrap-SRA Auvergne, 4 vol., $1717 \mathrm{p}$.
Conte P., Fau L., Hautefeullle F., 2010, « L'habitat dispersé dans le sud-ouest de la France médiévale ( $\mathrm{x}^{\mathrm{e}}$-XVII ${ }^{\mathrm{e}}$ siècles) », in CHAPELOT J. (éd.), Trente ans d'archéologie médiévale en France: un bilan pour un avenir, Actes du $9^{\mathrm{e}}$ Congrès international de la Société d'archéologie médiévale (Vincennes, 16-18 juin 2006), Caen, Publications du CRAHM, p. $163-178$

Dupuis M. et al., 2011, Combronde, les Cruchades, rapport d'opération, Hadès-SRA Auvergne, 3 vol.

Frere S., Hartley B. R., 1966, « Fouilles de Lezoux (Puy-de-Dôme) en 1963 ", Cahiers de civilisation médiévale, 9-36, p. 557-563.

Gabayet F. et al., 200o, Beaumont. Bassin d'orage aval de Pourliat. Lieu-dit la Mouraude, DFS, Afan-SRA Auvergne.

Gabayet F., Gauthier F., Horry A. et al., 2005, «Un établissement agricole du XIII ${ }^{\mathrm{e}}$ siècle à Beaumont, lieu-dit la Mouraude (Puy-de-Dôme) », Archéologie médiévale, t. 35, p. 97-139.

Gaime S., Carlier M. et al., 2016, Lezoux (Puy-deDôme), les Colis, route de Billom : genèse et transformations d'une occupation marginale sur le temps long, rapport d'opération, Inrap-SRA Auvergne, 3 vol. (1368 p.).

Gaime S., Gauthier F., Mille P. et al., 2011, «Un site castral bourbonnais au début de la guerre de Cent Ans : le Tronçais à Chevagnes (Allier) ", Archéologie médiévale, t. 41, p. 77-122.

Gaime S. et al., 200o, Pierrefitte-sur-Loire (Allier). Le bois Vernillat, DFS, Afan-SRA Auvergne.

GAIME S. et al., 2001, Billezois (Allier), Falconnière: les dépendances d'une maison forte au XIV siècle, DFS, Afan-SRA Auvergne, 2 vol. (58 + 61 p.).

Gaime S. et al., 2002, Clermont-Ferrand (Puy-de-Dôme). Croix de Neyrat. Poste Edf. «Une Occupation Médiévale : Cimetière et habitat ", rapport d'opération, Inrap-SRA Auvergne, 2 vol.

GAIME S. et al., 2005, L'habitat rural de Changy au $X I V^{e}$ siècle, rapport d'opération, Inrap-SRA RhôneAlpes, 2 vol.

GaIme S. et al., 2011, Chevagnes, le Tronçais, «motte, maison forte, pont-levis et forge ", les caractéristiques d'un site castral bourbonnais, rapport d'opération, Inrap-SRA Auvergne, 2 vol. $(328+590$ p.)

Gauthier F. et al., 200o, Puy Guillaume - la Cabane, DFS, Afan.
Gauthier F. et al., 2014, Riom, 4 Chemin des vignes, rapport d'opération, Inrap-SRA Auvergne.

Goy M. et al., 200o, Trévol (Allier), Les sites médiévaux de la Petite Perche, Route Nationale 7, DFS, Afan.

LIÉGARD S., Fourvel A., 2003, « Les habitats laténiens et carolingiens du site de "Bel-Air" à Lapalisse (Allier) ", Revue archéologique du Centre de la France, 42, p. 5-39.

LiÉGARD S., FourVEL A., 2010, « L'établissement rural des $\mathrm{IX}^{\mathrm{e}}$ - $\mathrm{X}^{\mathrm{e}}$ siècles des "Jeanrais" à Saint-Prix (Allier) », Revue archéologique du Centre de la France, 49, p. 233-282.

Lurol J.-M. et al., 1999, Chassenard (Allier), la Générie, DFS, Afan.

MéLOCHE C., 2015a, «Constructions médiévales en torchis. Étude de fragments issus de la démolition d'un bourg de la plaine du Doubs », Archéopages, $\mathrm{n}^{\circ} 42$, Construire en terre crue, p. 100-105

Méloche C., 2015b, Franche-Comté, Petit-Noir, Au Village, les abords d'un quartier médiéval (XIII ${ }^{e}$-XIV $V^{e}$ siècles), rapport d'opération, Inrap-SRA Franche-Comté, $228 \mathrm{p}$.

Mennessier-Jounnnet C., 1993, Ateliers de potiers gallo-romains, fours de potiers du haut Moyen Âge et bâtiment médiéval XII ${ }^{e}$-XIII à Lezoux. Lezoux, place de la République (Puy-de-Dôme), RFO, Afan.

Mennessier-Jounnnet C. et al., 2006, De l'âge du Bronze au haut Moyen Âge dans le bassin de Sarliève. Fouille au Carré du Bois Joli à Cournon-d'Auvergne (Puy-de-Dôme), rapport d'opération, Inrap-SRA Auvergne, 2 vol.

Pesez J.-M., 1986, « Le foyer de la maison paysanne ( $\mathrm{xI}^{\mathrm{e}}-\mathrm{XV}^{\mathrm{e}}$ siècles) », Archéologie médiévale, XVI, p. 65-92.

Sirot É., 2011, Allumer le feu, Cheminée et poêle dans la maison noble et au château du XII ${ }^{e}$ au XVI $I^{e}$ siècle, Paris, Picard, 184 p.

Surmely F., Franklin J., 2014, «Les Yvérats (Compains, Puy-de-Dôme) », Bilan d'activité 2014/Journée archéologique 2015, DRAC Auvergne, p. 61-62.

VogeL V., 1994, « De Haithabu à Schleswig », in Archéologie des villes dans le Nord-Ouest de l'Europe (VII ${ }^{e}$-XIII ${ }^{e}$ siècle), Actes du IV ${ }^{e}$ Congrès international d'archéologie médiévale (Douai, 26, 27, 28 septembre 1991), Caen, Société d'archéologie médiévale, 4 , p. $17-26$. 